\title{
El ferrocarril en El Salvador: de la ilusión al abandono en una breve cronología (1872-2018)
}

\author{
The railroad in El Salvador: from illusion to abandonment. \\ A brief chronology (1872 - 2018).
}

DOI: https://doi.org/10.5377/koot.v0i11.10733

URI: http://hdl.handle.net/11298/1189

Oscar Flores-Guzmán

Pasante de la Biblioteca Nacional de El Salvador

ooscarguzman@gmail.com

Fecha de aceptación: noviembre 2019

\section{Resumen:}

El sistema ferroviario surgió en el salvador como uno de los proyectos más ambiciosos en siglo XIX. Impulsado por el estado salvadoreño y por comerciantes de la época. El primer tramo de vías férreas en el país significó la apertura de la producción agrícola nacional a los mercados regionales, de Europa y EE. UU. Generó grandes transformaciones en todas las esferas de la vida social del país, pero a su vez este fue cohibido por las mismas fuerzas que lo impulsaban. A final de cuentas, terminó con más pena y con la añoranza de "lo que una vez fue", pero que no resta ni lo más mínimo a su relevancia histórica.

Palabras clave: Transporte ferroviario - historia - El Salvador. Ferrocarriles - historia - El Salvador - aspectos socioeconómicos. Trenes - historia - El Salvador. Locomotoras. Vías férreas.

\begin{abstract}
The railroad system began as one of the most ambitious projects in El Salvador during the XIX century. It was launched by the Salvadorean government and the businessmen of the period. The first section of the railway in the country meant the opening of the national agricultural production to regional markets in Europe and the U.S. It generated great transformations in different spheres of the social life of the country; however, it was held back by the same forces that drove it at the beginning. In the end, it ended up in sorrow and the longing of what "it once used to be;"notwithstanding, this does not take away any of its historical relevance.
\end{abstract}


Key words: Rail transport - history - El Salvador. Railways - history - El Salvador - socioeconomic aspects. Trains - history - El Salvador. Locomotives. Railways.

\section{Introducción}

El sistema ferroviario, cuyo ciclo de expansión-declive abarca los siglos XIX, XX y XXI de la historia nacional, surgió como uno de los proyectos más ambiciosos y fue impulsado por un estado salvadoreño "enamorado" de lo novedoso e imponente que resultaba y de los beneficios económicos, políticos e indirectamente culturales que este acarrearía a la nación, apoyado por comerciantes de la época que contemplaban en él, un medio de transporte que favorecería sus intereses particulares como productores agrícolas y comerciantes. Irónicamente, el germen temprano de aquel proyecto se hallaría en la tragedia sísmica de 1854 que destruyó la capital "obligando" a su traslado a la Hacienda Santa Tecla, y que -posteriormente- propició el cultivo de lo que sería el eje central de las exportaciones agrícolas venideras, focalizadas en gran parte en el cultivo del café, cuya movilidad se encarecía por la inexistencia de un medio de transporte adecuado para este fin, siendo el ferrocarril aquella solución.

Las influencias externas - a favor del ferrocarril- llegarían con la anexión de California a la Unión Americana y la fiebre del oro de 1848, engranada con el proyecto férreo de Panamá de 1855 que configuró un caldo regional que, exacerbado por la dinámica política, social y económica interna -por, sobre todo-, se declinó por la devoción al proyecto ferroviario a pesar de la escasa experiencia en planificación y gestión en este rubro. Devoción que desbordaría el ámbito económico propiamente dicho y que coexistió paralela e íntimamente relacionado con todas las esferas sociales a escala nacional, pero en esta ocasión la atención se centra en fechas concretas de gran relevancia para el desarrollo del ferrocarril en el país, como se verá más adelante.

El aspecto que dio un giro definitivo en pro de aquel sistema de transporte fue el cultivo y exportación del café a los mercados de EE. UU y Europa, con tendencia al alza a inicio de la década de 1860 a causa del declive paulatino en la producción y exportación de otros productos agrícolas como el añil. Por tanto, Entender el desarrollo del ferrocarril en El Salvador, requiere hacer énfasis en aspectos relevantes de esta empresa, como las contratas, tramos a explotar y demás. Pero que, en esta ocasión, se condensa en una cronología que acontecimientos históricos que proporciona luz sobre la importancia del gran sistema ferroviario nacional. 


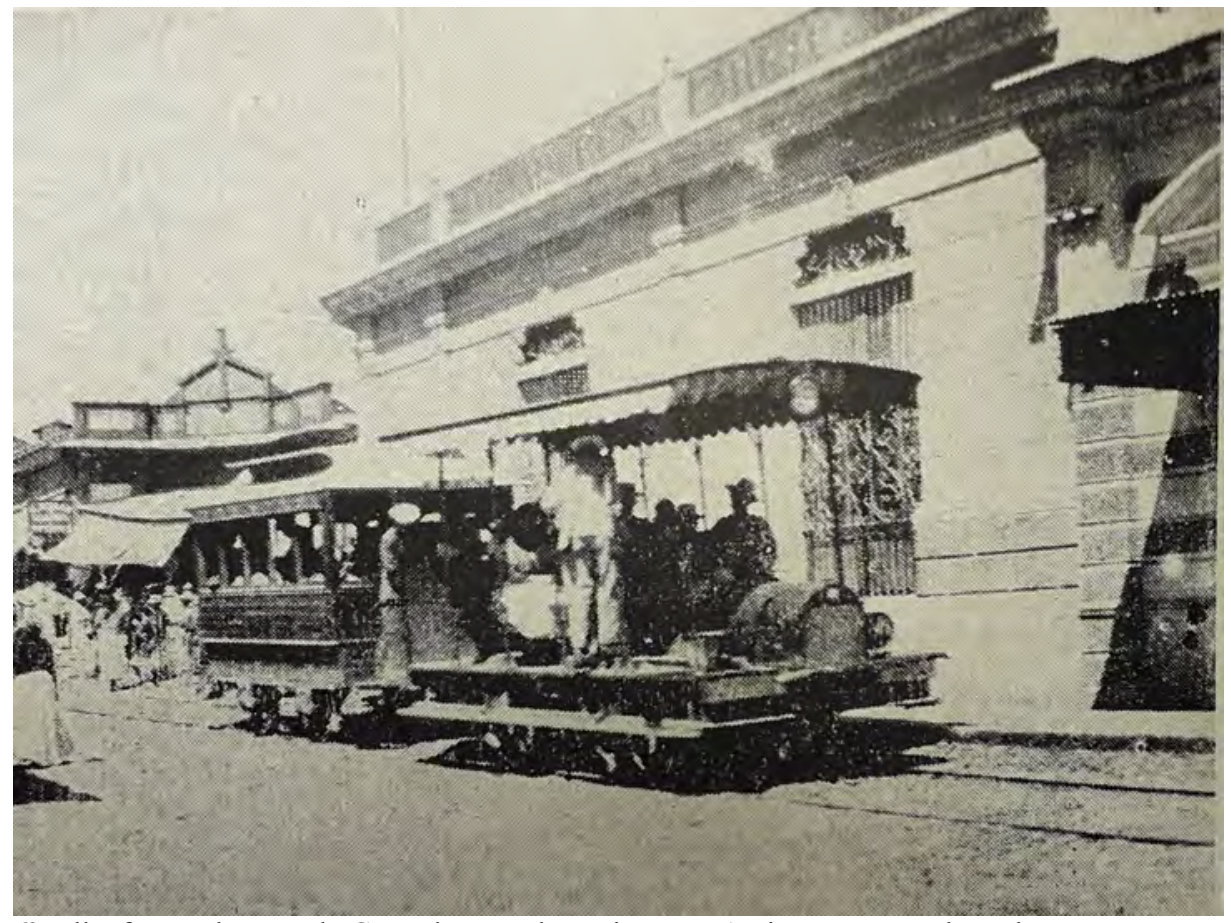

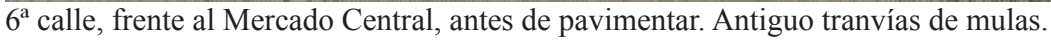

Fotos extraídas del libro: La obra de gobierno del doctor Quiñónez-Molina 1923-1927, tomo I.

\section{El Contexto social y el germen del sistema ferroviario como proyecto de nación.}

El terremoto del 16 de abril de 1854, que dejó en ruinas gran parte de la infraestructura de San Salvador, llevó el coronel José María San Martin -presidente de El Salvador para ese momento- a dictar una serie de decretos para que la capital se trasladara a Cojutepeque, "mientras se edificaba la nueva capital en los terrenos de la Hacienda Santa Tecla" (Martínez, 2017, p. 78). Según Chicas Molina (2015) el traslado de la capital se dio a un nivel legal más no en términos funcionales, ya que no se llevó a cabo un traslado formal de esta hacia Santa Tecla, ubicada a 15 kilómetros al poniente de S.S. En tanto las consecuencias del terremoto, fueron publicadas en el diario oficial La Gaceta núm. 55 del 19 de mayo de 1854, impreso en la ciudad de Cojutepeque -de forma inmediata luego del terremoto de abril de aquel año (p. 70). El gobierno lamentaba aquella tragedia y el desconcierto en el despacho de los negocios públicos [...que generó] la falta de antecedentes, de oficinas, de empleados, de localidades aparentes [...] que facilitan la expedición del trabajo gubernamental. Dejando claro que esta fue la razón que llevó a su traslado, pero a raíz de aquella tragedia. 
Era necesario pública y simbólicamente que el corazón de la república retornara a su sitio. Aquel importante evento llegaría el 28 de julio de 1858, por acuerdo ejecutivo número 5, se mandó a que el Gobierno del estado salvadoreño -con el General Barrios a la cabeza- se trasladara a la antigua ciudad de San Salvador y delegaba a Cayetano Bosque -ministro general de Gobierno- de lo "conveniente para la conducción de todos los enseres que pertenezcan a la Administración, así como los archivos [relativos al quehacer de la gobernanza estatal...]" (García, 1954, p. 10). Sin embargo, lo relevante del traslado y posterior retorno de la capital fue que estableció un hito territorial, aumentó la plusvalía de las fincas de café aledañas -cuyo grano se presentaba como eje central en el desarrollo urbano venidero de San Salvador- (Chicas Molina, 2015, p. 70) y dio un impulso cualitativo al cultivo extensivo del café mismo.

En este sentido, resultaría trascendental la figura de Rafael Campo quien en 1856 -como presidente de El salvador- considera que los terrenos de la Hacienda Santa Tecla debían ser destinadas a la agricultura -con énfasis en el cultivo del café- y cuya superficie debía ser repartida en solares de seis y veinte manzanas para aquel fin, pero expropiados en caso de que los poseedores de aquellos terrenos no los utilizaran para el cultivo del cafeto (Leistenschneider, 1980). A pesar de la intuición de Rafel Campo, el cultivo del café -para ese momento no se había posicionado por sobre la producción de añil, aunque este último ya estaba en un proceso de recesión -parcialmente- a causa de la incapacidad para competir con los precios a que se comercializaban los tintes sintéticos que surgieron luego de la Revolución francesa de finales del siglo XVIII.

A partir de la década de 1860 las exportaciones de añil empezaron a decaer ante el nuevo cultivo de importación, a saber: el café, que se posicionaba con fuerza. Sin embargo, las características propias del "grano de oro" -con énfasis en su peso- encarecían los fletes de transporte a causa de las dificultades que tenía su movilidad. Hasta ese momento las cargas se transportaban a "lomo de bestia", donde la carga de añil se favorecía por su escaso peso en relación a su masa pero que, a diferencia con el café, lo dificultaba y encarecía sus costos de movilidad hacia los principales puertos nacionales y regionales para su comercialización dado su mayor peso en relación con la cantidad en masa, lo cual "creó" -en parte- la necesidad de buscar un medio de transporte que facilitara aquella tarea que, después de satisfecha, daría un impulso incuestionable al comercio nacional con vista a la región centroamericana y sociedad global con énfasis en el marcado estadounidense y europeo (Lindo Fuentes, 2002).

En respuesta, el ferrocarril se sugería como una opción novedosa, pero impulsado en buena medida por influencias externas. Con la anexión de California a los EE. UU. y la "fiebre del oro" que arranca por un hito de casualidad ese mismo año en los terrenos anexos a la Unión Americana, la cual se encontraba en plena expansión 
en todos sus frentes, generó un aumento en el flujo de personas y de comercio hacia su costa del Atlántico -por encontrarse allá, aquellas minas-, abonado por la puesta del ferrocarril en Panamá para 1855 y su servicio de vapor -proporcionado por la Pacific Mail Steamship Company, popularmente identificada en el país como "las malas del Pacifico"- evocó un aumento y una estabilidad en la diligencia de los puertos de La Unión, La Libertad y Acajutla, lo cual regularizó el servicio de estos y facilitó el ingreso de las importaciones, mientras que las exportaciones agilizaban la partida a sus destinos, consolidando, a su vez, nuevas rutas de transporte (Ministerio de Educación [MINED], 2009, p. 28).

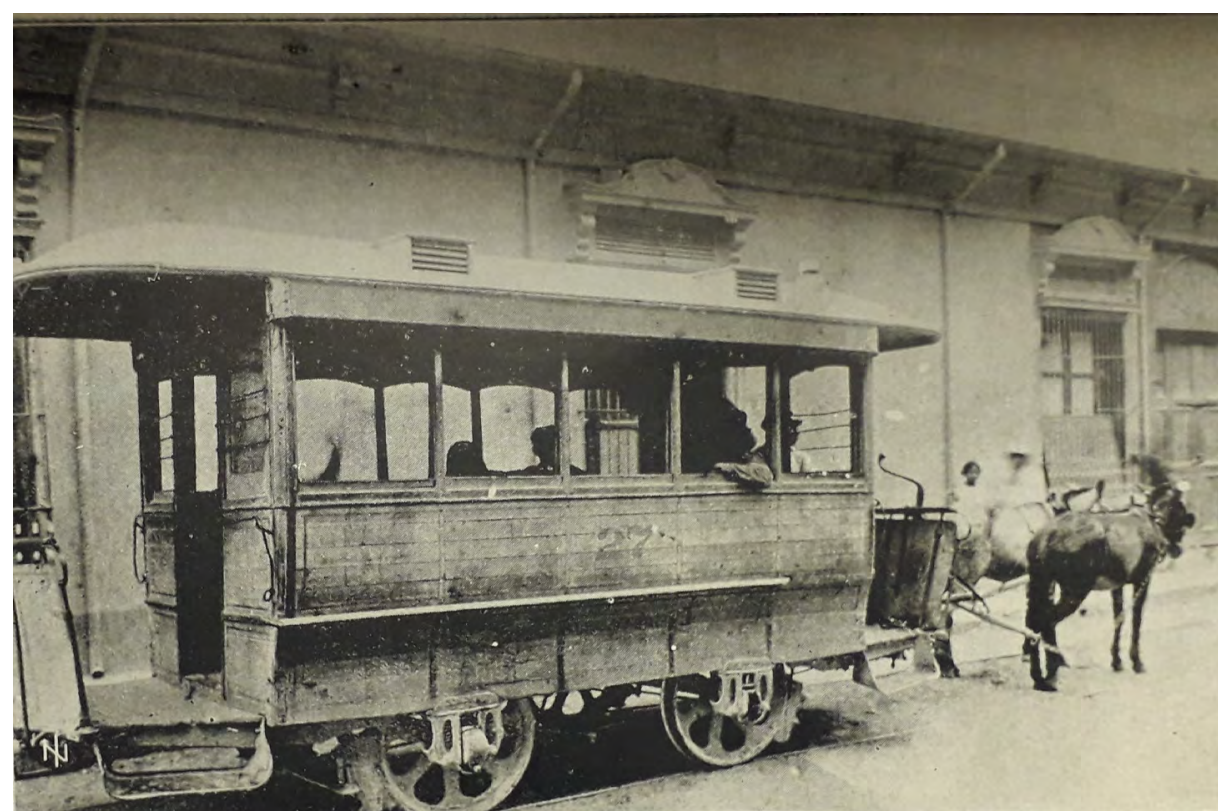

Vista del antiguo medio de transporte en El Salvador.

Fotos extraídas del libro: La obra de gobierno del doctor Quiñónez-Molina 1923-1927, tomo I.

\section{El ferrocarril: de la expansión al declive, 1872-2018}

Las nuevas rutas comerciales necesitan un sistema de trasporte coherente a las nuevas exigencias de los mercados y sus productores y el ferrocarril iría encaminado a este fin. Según Morrison (2009), en alusión a los antecedentes más tempranos de este medio de transporte a escala nacional, se hayan en la administración del mariscal de campo Santiago González Portillo, quien para "1872 otorgó a Jean-Louis Bueron (o Juan Büron)" -de nacionalidad francesa pero nacido en Prusia- la contrata para construir las líneas férreas que conectarían los Puerto de Acajutla, La Unión y La Libertad y propuso que el primer tramo, conectaría San Salvador y Santa Tecla, el 24 de junio de 1873. 


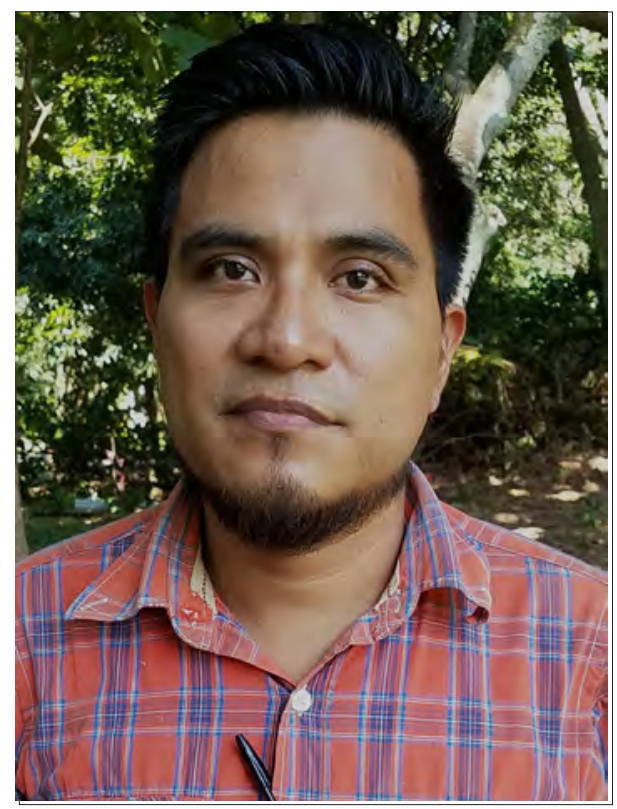

Oscar Flores Guzmán

Al respecto de esta contrata, el Boletín Oficial del 25 de septiembre de 1872 incluyó tres publicaciones íntimamente relacionadas. En una de ellas el presidente González Portillo manifestaba su gratitud al Sr. Bueron -dado que este sería el encargado de ejecutar el primer proyecto ferroviario en el territorio salvadoreño y por las dadivas que este entregó -luego de ingresar al país procedente de Europa- una "fina" espada, una bomba apaga incendios y un báculo que fueron entregadas al Sr. presidente, a la municipalidad capitalina y al arzobispo Miguel Pineda i Saldaña, respectivamente-. El jefe de estado consideraba que el proyecto que sería ejecutado por el Sr. Bueron significaría la apertura al progreso nacional (Comisión Ejecutiva Portuaria Autónoma, s. f.).

En el mismo tiraje, el señor Álvaro Contreras -redactor del Boletín Oficial de El Salvador en aquel momento, aunque de origen hondureño- señalaba que: aquellos trabajos significaban la lucha del capital contra la naturaleza. Consideraba también, que el proyecto ferroviario generaría cuantiosas ganancias y que, sus líneas, serían favorecidas dada su ubicación geográfica pues permitiría que estas fueran beneficiadas por las líneas interoceánica establecida en los países vecinos.

La ilusión que proponía aquel proyecto sufriría lapsos de abandono y recelo, resultado de la inexperiencia en gestión y planificación de este tipo, sin embargo y a pesar de esto, los gobernantes de la época se veían ilusionados por lo atractivo y novedoso de aquella iniciativa. Por otro lado, el mismo 
Bueron no previó las dificultades que este podía tener en sí. Ejemplo de esto fue el sismo de marzo de 1873 que causó severos daños a la ciudad, generó un clima de incertidumbre por parte de los inversionistas, y lo llevó a solicitar la modificación del contrato original, "trastocando los plazos de entrega (a seis años) y el porcentaje (8\%) que le correspondía en forma de garantía a favor del empresario por sobre el costo de las vías tras su explotación, quedando nulo este punto en caso de que estas dejasen de funcionar por algún motivo (Trujillo, 2015, p. 69).

Tres años serían necesarios para que el tramo inicial se concluyera, Según García (1954), el magno evento tuvo lugar el 27 de julio de 1876 a las 8:30 a.m., hora a la que arribó el primer tren -o tranvía- proveniente de Santa Tecla recorrido de ida y vuelta que tenía un costo de tres reales o se podía adquirir billetes de abono de 10 colones (hoy \$1.15), válidos para cincuenta viajes-. Aquel vagón conducía al alcalde, gobernador civil y al comandante general militar mientras que el Dr. Zaldívar y su gabinete aguardaba para recibirlos, el vagón era empujado a fuerza animal -o de sangre- pero guiado por el infatigable Sr. Bueron. (pp. 461-462).

La misma fuente sugiere que el financiamiento para consumar aquella obra provenía -en parte- del sector gubernamental y de comerciantes, como el $\mathrm{Sr}$. Miguel Lagos, que motivados por la necesidad de ver terminados aquellos trabajos, facilitaron los fondos que fuesen necesarios para tal fin (p. 461). Por su parte, el acreedor de aquella explotación -y antes de su inicio- debió realizar un viaje a Francia para concretar una compañía que lo respaldara y para lo cual el gobierno le facilitó buenas garantías para entregar tras concluir dichas vías -garantías que servirían de motivación a posibles inversores en apoyo a Bueron(Trujillo, 2015).

Sin embargo, la contrata inicial impedía realizar nuevos pactos para próximas explotaciones a raíz de cláusulas que favorecían al Sr. Bueron, que impedían la competencia y daban el beneplácito para la explotación de los bosques circundantes a las vías férreas (Martin, 1985), pero el aumento en el tráfico de personas y comercio, vinculado a la incapacidad para satisfacer aquella alza, llevó a gobierno a cabildear nuevas concesiones para tal fin (Solorzano, 1977). Así, el 29 de noviembre de 1881, según FENADESAL (2016), el gobierno salvadoreño y la compañía Ferrocarriles de Acajutla suscribieron un contrato para la explotación del tramo que iría del Puerto de Acajutla a la Ciudad de Sonsonate -aunque el acuerdo se hizo oficial hasta marzo de 1882. 


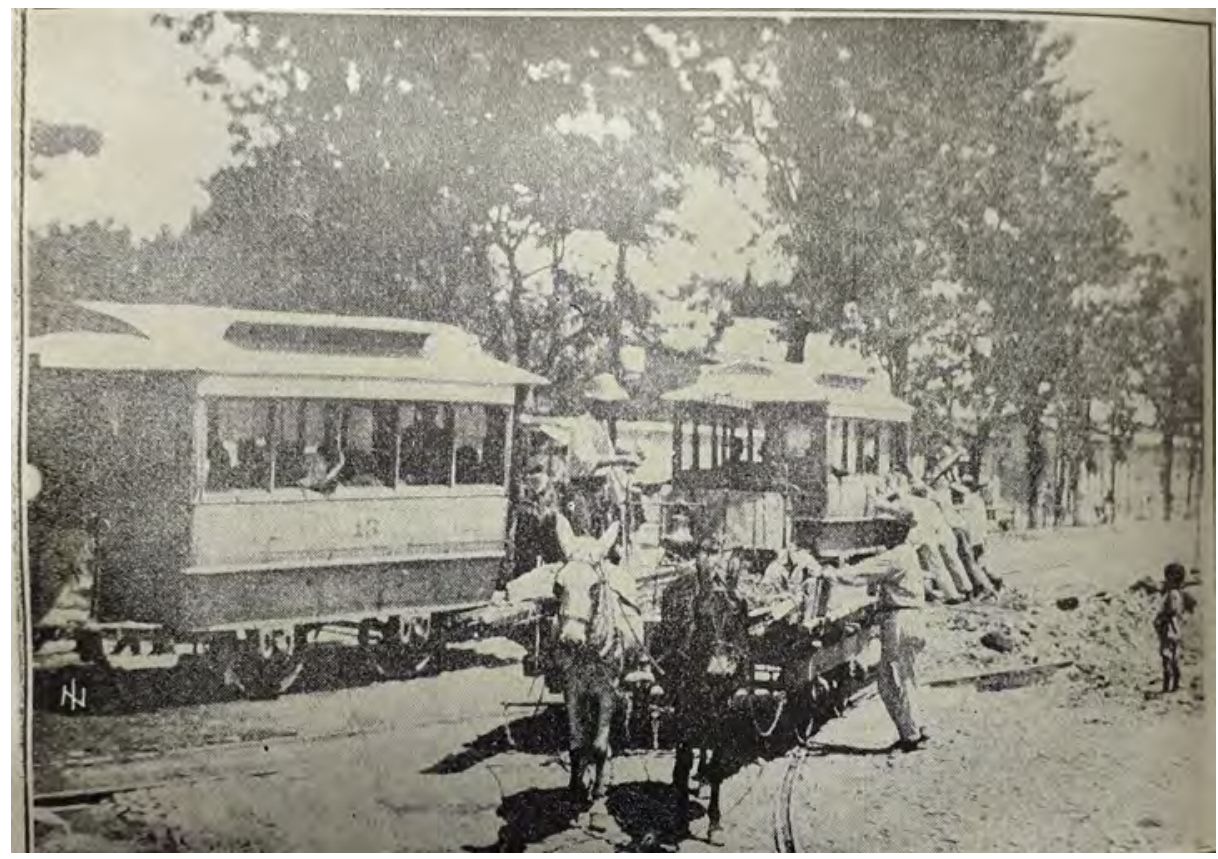

Avenida Independencia, antes de la pavimentación. Un cambio de tranvía.

Fotos extraídas del libro: La obra de gobierno del doctor Quiñónez-Molina 1923-1927, tomo I.

Otras fuentes (Martin, 1985), sugieren que en este mismo periodo se realizaron otras contratas que dieron paso a que el sistema ferroviario, en el Occidente del país, se desarrollara de la siguiente manera: el primer tramo fue el de Sonsonate-Armenia, con una distancia de $26 \mathrm{~km}$., posteriormente se sumarían $6.5 \mathrm{~km}$, agregándose $3 \mathrm{~km}$, llegando a Ateos. De este punto se extendió otros 22 kilómetros, hasta llegar a la ciudad de Santa Ana -este último segmento inaugurado en noviembre de 1896-, acumulando una distancia de $51 \mathrm{~km}$. Siendo esta última ciudad la terminal donde el ferrocarril recibía "su apreciable carga consistente en productos agrícolas, tales como café, azúcar, tabaco y varias clases de granos" (p. 202).

La compañía: Central American Public Works Company Limited -propiedad de Mr. Scherzer- sería la encargada de completar el tramo de Ateos a Santa Ana, continuando así los trabajos ferroviarios al Oriente del país, corría el año de 1884 cuando se acordó con dicha compañía aquellos trabajos (Solorzano, 1977). Sin embrago, lo relevante de este contrato es que abrió camino para que la compañía británica The Salvador Railway Company Limited -posteriormente conocida como Ferrocarril de El Salvador (FES)- se asentara en el país, bajo la figura legal de "concesión" que la American Central se guardaba en el apartado 
de 28 de su contrato y en acuerdo con el supremo gobierno se llevó a cabo aquella licencia firmada en Londres, Inglaterra en 1897 por The Salvador Railway (pp.42).

Según Martin (1985), el compromiso con la compañía The Salvador Railway Company Limited, fue que poseería un período de legalidad de 80 años. Luego de vencer este ciclo el ferrocarril y sus accesorios pasarían a ser propiedad del Estado. Este último, por su parte, se reservaba el derecho de adquisición de aquel sistema en 1940 a un precio fijado luego de una evaluación y a merced de cualquier eventualidad que llevase a finiquitar el pacto entre la compañía y el Estado salvadoreño antes del plazo consignado.

Aesta "altura", resulta incluso necesario cuestionar cómo el estado proporcionaría los terrenos que necesitaría el proyecto ferroviario. En parte queda resuelto con la publicación del Diario Oficial del 13 diciembre de 1895, donde se anunció un decreto firmado por Rafael Gutiérrez -presidente de la República- y Prudencio Alfaro -secretario de Estado en el despacho de gobernación-, el cual brevemente reza de la siguiente manera:

Tomando en consideración que es de utilidad pública la continuación de la línea férrea hasta el puerto viejo, y que para ellos se necesita ocupar algunos terrenos de particulares en jurisdicción de Acajutla [...]. Decreta: Declárese de utilidad pública la ocupación de los referidos terrenos y procédase á su expropiación en la forma legal [sic] (p. 1917).

En esta ocasión, no se halló evidencia que sugiriera las indemnizaciones que -posiblemente- otorgaría el mismo gobierno a aquellos particulares afectos por el decreto publicado en esta fecha.

El 16 del mismo mes y año, pero publicado el 30 de diciembre en el Diario Oficial -núm. 306-, tuvo lugar la inauguración de los trabajos para la construcción del tramo que uniría La Unión -ubicado al oriente del país- con la capital y que, según esta publicación, estaría a cargo de un cuerpo respetable de ingenieros cuya comisión llegaba de Londres, aunque sin más detalles del currículo del equipo de ingenieros. Por su parte el presidente Gutiérrez, señaló en aquella ocasión que, ya que el occidente y el centro del país pronto, para aquel año, estarán interconectados por ramales de vías férreas, resulta de vital importancia que el oriente se incorpore al concierto del desarrollo nacional que traerá consigo el proyecto ferroviario.

Entre los asistentes que disfrutaron de un espacio para su alocución estaba Rafael S. López -representante del gobierno-, quien consideraba: que el complejo proceso de interacción entre factores materiales, morales e intelectuales 
daría como resultado el progreso nacional y que el abandono de uno de ellos vuelve incompleta y defectuosa la obra. El Dr. Antonio Rosales -representante departamental de San Miguel- aseguraba que: los trabajos inaugurados aquel día, facilitaría la comunicación del puerto de Cutuco -en La Unión- con los principales centros comerciales de la república, para el engrandecimiento de la sección Oriente y de toda la Nación (p. 1969).

Importantísima obra que necesitaría una 'buena dosis' de mano de obra que permitiera consumarla de buena manera. El telegrama enviado al señor presidente Rafael Gutiérrez- por G. Quiróz el 31 de diciembre de 1895 -publicado en el Diario Oficial de aquella fecha- dejaba entrever que para realizar los trabajos recién inaugurados trabajaron 400 operarios de sexo masculino que -según el remitente- en su mayoría eran voluntarios, pero a causa de cambios administrativos a nivel local se generó una disminución en el número de trabajadores, aunque -para ese momento- ya se había concluido una milla para su enrielado.

Según Martin (1985) en 1908 tiene lugar una nueva concesión -conocida como contrata Avalos-Keilhauer- para construir el tramo que conectaría desde La Unión hasta la frontera con Guatemala. Este último ramal pasaría a ser el vínculo entre las ciudades de Usulután, San Vicente y Cojutepeque e interconectaría, también, con el tramo que iba de San Salvador hacia Santa Ana, desembocando con el ferrocarril del Atlántico. La importancia de este último tramo es que abarcaría la sección de la línea Panamericana al interior del territorio nacional. La contrata de 1895, para construir la línea férrea de La Unión a San Miguel tendría su fruto para el año de 1912. El 6 de julio de ese año, el Diario Oficial realizó la publicación alusiva a la inauguración de aquel tramo -inaugurado por el entonces presidente Manuel Enrique Araujo- quien pronunció un elocuente discurso -publicado en este número- donde aseguraba que dicha obra abría: "[...] aquella rica sección de la patria salvadoreña, [a] una nueva era de prosperidad [...y] progreso. San Miguel, la metrópoli de Oriente, [...] renace á nueva vida, y la savia de nuevas energías, hará que allá florezcan la agricultura, el comercio y las industrias (sic)" y dejaba entrever el deseo de continuar los trabajos hasta las riberas del río Lempa (p. 1517).

En 1912, con el ingreso de la compañía Ferrocarriles Internacionales de Centroamérica, y luego de varias dificultades económicas, se logró iniciar operaciones en el tramo que comprendía entre el Puerto de Cutuco (La Unión)San Miguel- y luego a San Salvador para 1920 (CEPA, 2014, p.43). Cabe la aclaración, de que los derechos y obligaciones contraídos por el IRCA tenían su antecedente en la contrata Avalos-Keilhauer pero que su concesionario -René Keilhauer- cedió los derechos de la contrata original a la compañía Guatemala Railway Company conocida después como International Railway of Central 
America -conocida como IRCA por sus siglas en inglés- con domicilio en el estado de Nueva Jersey, EE. UU. (Solorzano, 1977).

Para 1927, aproximadamente, esta compañía cruzaba

casi todo el territorio salvadoreño. Actualmente [para el mismo año] está en explotación desde el puerto de Cutuco (La Unión) hasta la capital: 252 kilómetros. El tren pasa por los departamentos de San Salvador, Cuscatlán, San Vicente, La Paz, Usulután, San Miguel y La Unión [dejando entrever las nuevas rutas en explotación, por ejemplo:] la línea de Soyapango a Texistepeque [... y] los ramales de Santa Ana a la frontera de Guatemala (Andino, 1927, p. 24).

En 1962, a causa de incumplimiento de contrato por parte de la The Salvador Railway Company Limited -compañía que había hecho saber al estado salvadoreño su incapacidad para administrar el sistema ferroviario a su cargoesta había acordado hacer el traspaso de forma legal de todos los bienes muebles e inmuebles del ferrocarril a su cargo en favor del gobierno nacional (Solorzano, 1977). Por tal razón, y

tomando en cuenta el abandono total que había hecho del aludido servicio público [la compañía en cuestión], [...] se llegó a la resolución de solicitar al Poder Legislativo la declaratoria de la caducidad de la concesión otorgada a la The Central America Public Works Limited y cedida por esta a The Salvador Railway Company Limited. (pp.48-49).

A raíz de lo anterior, se promulgó el decreto legislativo núm. 181 del 22 de octubre de 1962, mediante el que se constituye el Consejo de Administración del Ferrocarril de El Salvador y para 1971 el IRCA se declara en quiebra y pasa a ser administrada por el estado (CEPA, 2020, p. 6). El 3 de octubre de 1974 el gobierno declaraba caduca la contrata Avalos-Keilhauer y demás modificaciones que sobre esta se habían realizado a causa de incumplimientos en las cláusulas contractuales. Fue a partir de 1974 que se delegó a la Comisión Ejecutiva Portuaria Autónoma (CEPA) la dirección, operación y administración tanto del sistema ferroviario como del portuario, conociéndose desde ese momento como: Ferrocarriles Nacionales de El Salvador (FENASAL) (Solorzano, 1977). El año siguiente, por Decreto Legislativo núm. 269, publicado en el Diario Oficial del 22 de mayo de 1975, las compañías: Ferrocarriles de El Salvador (FES) y FENASAL se fusionan y nace Ferrocarriles Nacionales de El Salvador -FENADESAL-. Aquel decreto contemplaba que, en vista de que las empresas a cargo del ferrocarril y del puerto de Cutuco -cuyas concesiones yacían nulaspasarían al pleno dominio del estado sus propiedades y dependencias. Al Fusionarlas se buscaba prestar un servicio coordinado entre el ferrocarril y el 
puerto de Cutuco, lo que permitiría ejecutar una política coherente y encaminada a armonizar el comercio terrestre y marítimo, respondiendo solo a los intereses propios de la nación y para efecto de todos los trámites administrativos, siempre estaría subordinada a CEPA.

Por tanto, rezaba el artículo 1: "fusiónanse (sic) las empresas ferrocarrileras estatales identificadas como Ferrocarriles de El Salvador (FES) y Ferrocarril Nacional de El Salvador (FENASAL), incluido en esta última el Puerto de Cutuco. Las empresas que se fusionan integrarán el sistema nacional de ferrocarriles de El salvador en una solo empresa, cuya denominación será Ferrocarriles Nacionales de El Salvador o abreviadamente FENADESAL" (p. 5727).

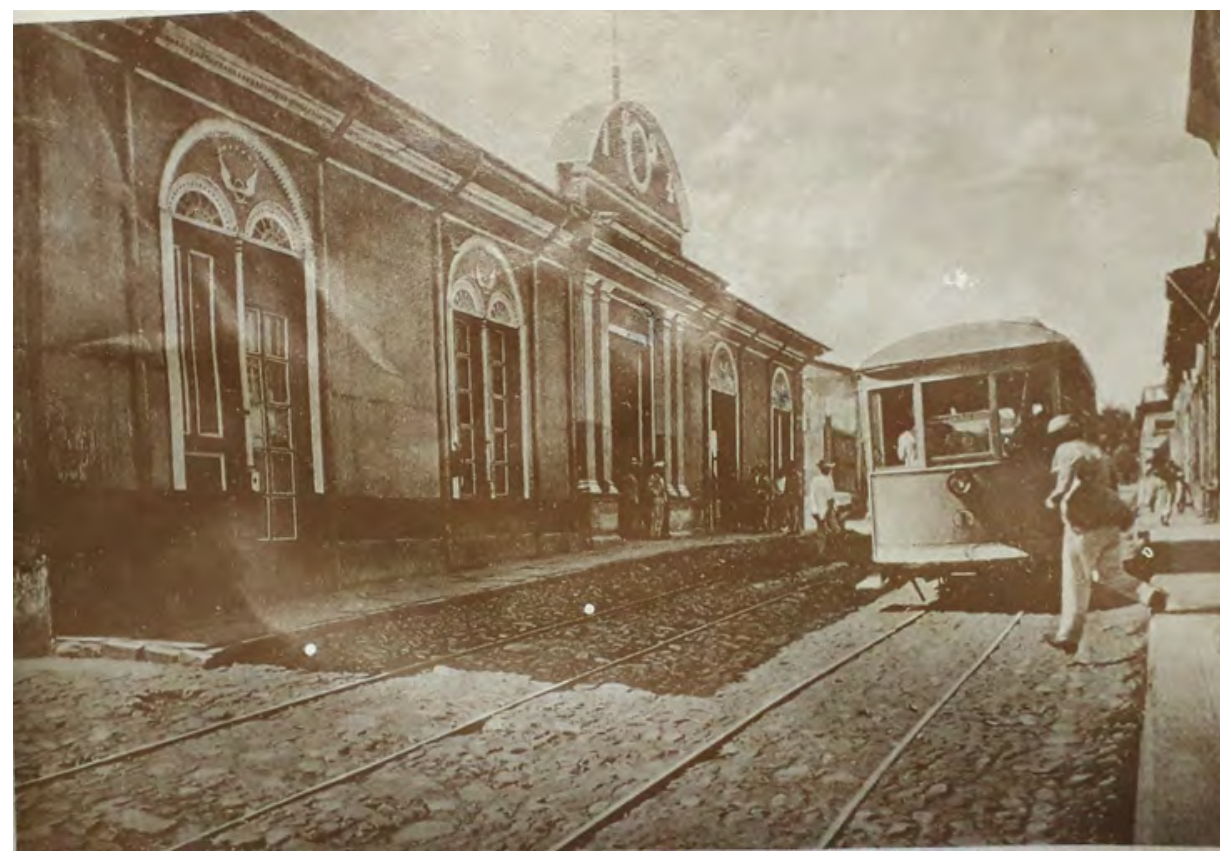

Hotel Hispanoamericano, en la esquina de la $4^{\mathrm{a}}$ Calle poniente y $2^{\mathrm{a}}$ avenida sur, hoy en toda esta manzana se encuentra el Banco Hipotecario Central.

Fotos extraídas del libro: Paseo del recuerdo. San Salvador 1900-1925.

Luego de la fusión de las compañías ferroviarias, según CEPA (2014), las operaciones no se detuvieron, registrando una leve mejora en el rubro de carga -en términos de peso transportado- para 1976 (460, 897 t., comparada con el año base: 1951 en esta referencia), superando para 1979 en $15 \%(589,497$ t. / 2,010,574 pasajeros) a las estadísticas en este rubro de 1951 (516,000 t. / $3,431,000$ pasajeros), pero por otra parte el transporte ferroviario decaía ante la preferencia de la población por el transporte terrestre. Particularmente, al 
finalizar el conflicto político-militar -tras la firma de los Acuerdos de Paz el 16 de enero de 1992, en el Castillo de Chapultepec, México- inició un período de reconstrucción.

La empresa cargaba con dos enormes lastres: el daño físico, incluyendo el deterioro de infraestructura por la falta de mantenimiento y renovación de sus equipos, más el descenso secular en el uso del ferrocarril como medio de transporte de pasajeros y carga debido a la competencia [con el] tráfico vehicular (p.50).

Aldana (1997) se refiere a que, aproximadamente al año de su publicación y durante la administración presidencial de Armando Calderón Sol (19941999) se generaba una gota de confianza porque -el ejecutivo- revitalizara FENADESAL, retomando los ofrecimientos de colaboración por parte de países como Japón, Francia, Inglaterra, Italia y otros con el ánimo de colaborar con la modernización del sistema ferroviario nacional, pero que habían sido ignorados hasta ese momento.

Según este autor, para aquel año las vías coincidían con las que una vez inauguraron (The Salvador Railway Company Limited - IRCA) a finales del siglo XIX e inicio del XX. La rehabilitación sería un largo y complejo proceso y que necesitaría del soporte de gobiernos "amigos" que apoyen aquella iniciativa.

Japón se perfiló en este sentido -por el apoyo que venía siendo ofrecido desde 1962 a 1967- y aunque hubo intercambio de ideas no se llegó a acciones concretas a raíz de intereses obscuros que lo imposibilitaron. Señalaba en parte -con una buena dosis ideológica de post-Gguerra Fría- las décadas de agonía del ferrocarril nacional -antes, durante y después del conflicto político-militar- a raíz del terror que causó aquel trance bélico que envolvió al país desde -al menos- 1980. Pero que, su rehabilitación a finales del siglo XX resultaba necesaria "no porque [.] signifique una de las mejores obras que dejaría el actual gobierno, [de Calderón Sol] sino por lo que representaría para la posteridad" (p.25-26).

A pesar de las "buenas intenciones" encaminadas a la reactivación del ferrocarril en el país, ninguna alcanzaría la "gloria" que -primitivamente- gozó el sistema ferroviario. En el 2002, el que una vez fue proyecto símbolo de modernidad y progreso para la nación, dejó de prestar servicio, indemnizó a sus empleados y redujo su personal únicamente al necesario para su administración y seguridad. Sin embargo -y a pesar del trágico 2002- en el año 2007, desde CEPA se tomó una importante decisión en un intento de revivirlo, pero con una estrategia diferente y ambiciosa: se descartó la revitalización del viejo sistema de trenes -disponible para FENADESAL en ese momento- y se buscaría la construcción 
de un nuevo sistema de vías -para carga y pasajero- que permitiera, luego de estandarizar la flota de trenes, anexarlo al sistema ferroviario de Guatemala y posteriormente interconectarlo con el sistema ferroviario de Norteamérica en el contexto del CAFTA -firmado por El Salvador en 05 de agosto de 2004 y ratificado el 17 de diciembre del mismo año- y del Plan Puebla Panamá -al que El Salvador se incorporó en el 2004- (CEPA, 2014, p. 53).

Finalmente, se registró un breve periodo en el que funcionó el ferrocarril -del impulso de 2007 hasta agosto de 2012- año en el que tuvo lugar su último recorrido. Este lapso se vio opacado por los altos índices de inseguridad social a escala nacional, aspecto que en parte fue responsable del rápido declive de aquel intento, posteriormente, en 2015 -bajo la administración como director de CEPA del Ing. Nelson Vanegas- se fundó el Museo del Ferrocarril y el Parque Temático que actualmente presta servicio en las instalaciones de FENADESAL, ubicados sobre la Avenida Peralta Núm. 903 -en las cercanías de la exterminal de Oriente en San Salvador (CEPA, 2016).

En esta línea, se han abierto otros espacios para la difusión de la historia del ferrocarril -como el Museo del Ferrocarril en Sonsonate, la Casa de la Cultura y Convivencia dentro de las instalaciones del Museo del Ferrocarril en san salvador - que busca propiciar "un espacio para la promoción del arte, la cultura y la participación ciudadana a los habitantes de las comunidades de la zona" (CEPA, 2018, p. 71), Dichas iniciativas, están lejos de aquellas ilusiones con las que inició el vaivén de uno de los proyectos más importantes de la historia salvadoreña, por transcender ampliamente su función de "transporte de carga y de pasajeros".

\section{A manera de conclusión}

A casi 147 años de la contrata para construir el primer tramo de vías férreas, en El Salvador, este significó, coordinadamente con el sistema portuario, la apertura de la producción agrícola nacional a los mercados regionales, EE. UU. y Europa. Generó grandes transformaciones en todas las esferas de la vida social, pero a su vez este fue cohibido por las mismas fuerzas que lo impulsaba y el milieu de su desarrollo jugaría su papel en el devenir de este ambicioso proyecto que, a final de cuenta, terminó con más pena que gloria y con la añoranza de "lo que una vez fue", pero que no le resta ni lo más mínimo a su relevancia histórica. Relevancia que se debe alejar de las idealizaciones románticas, para develar las contradicciones que aquel proyecto podía y puede contener en sí, todo con el único objetivo de tomar las medidas necesarias para una verdadera revitalización del sistema ferroviario nacional y - aunque no es punto para tratar en esta ocasión- aunque estas "cruzan" trasversalmente toda su historia. 


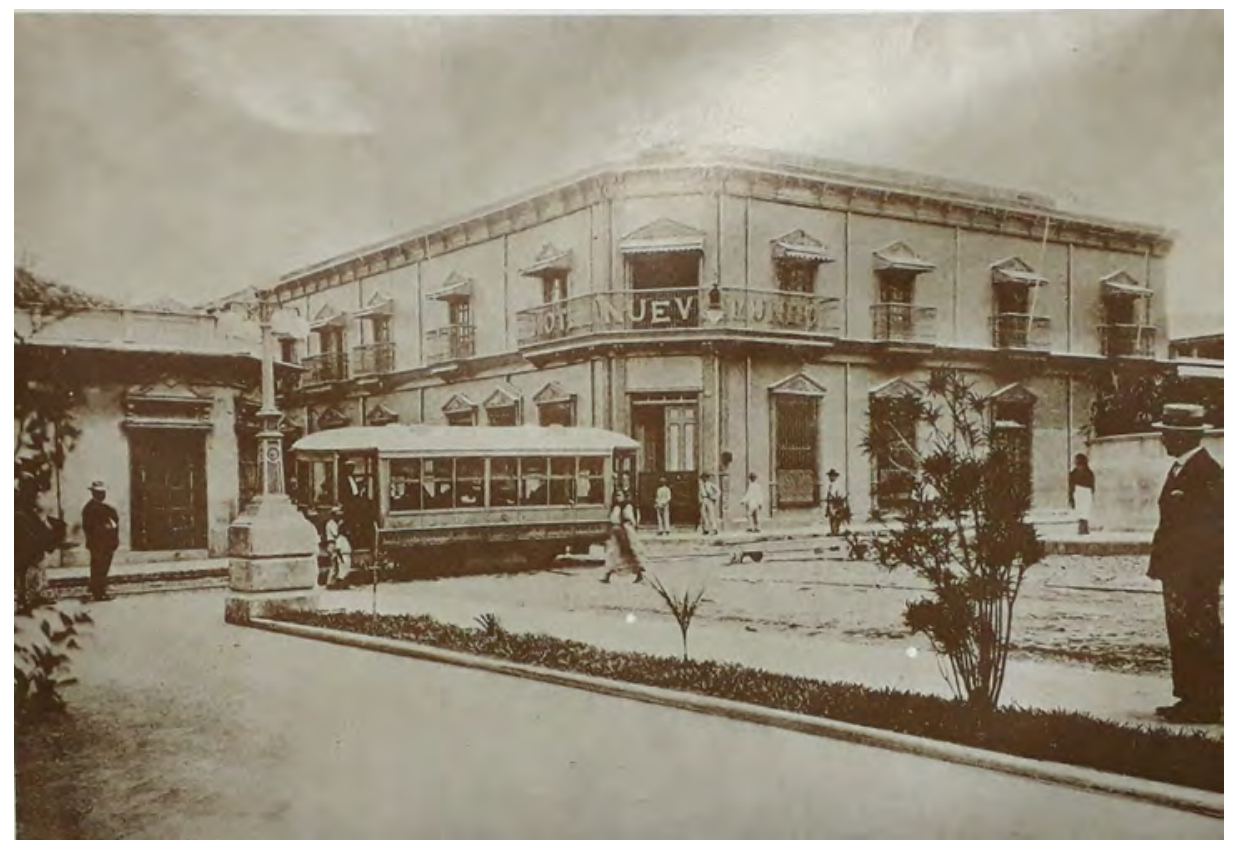

Hotel Nuevo Mundo 50 del Sr. Porth. Este lugar lo ocupó después el Hotel Astoria, y luego el almacén Vaciana; actualmente hay muchas tiendas comerciales. Esta vista tomada desde el

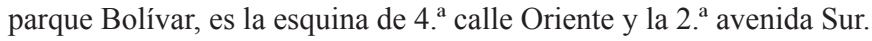

Fotos extraídas del libro: Paseo del recuerdo. San Salvador 1900-1925.

La paradoja resulta por demás interesante: aquel proyecto que ilusionó a toda la sociedad terminó convertido en piezas de museo que únicamente evocan la gloria que una vez contempló, pero -como se dijo más arriba- no le resta en lo más mínimo la importancia que este ejerció para el desarrollo del país. Un párrafo que, a pesar de lo extenso, resulta concluyente asegura que

[...] la red misma no se amplió desde el momento en que se completaron los planes originales allá por 1930. [...] el ferrocarril quedó congelado en el tiempo y no pudo adaptarse a los requerimientos de los usuarios y a los cambios en el tamaño y la distribución de la población del país. [...] "Era obvio que la modernización de la red ferrocarrilera pasaba por una importante inversión en compra de equipo rodante, rieles y sistemas de comunicación, además del mantenimiento de la infraestructura aprovechable (tales como puentes, viaductos y estaciones). De lo contrario, sería imposible competir con la opción del transporte automotor"' (CEPA, 2014, p. 52).

Aún hoy el ferrocarril aguarda, quizá, a un nuevo tiempo, una nueva reinvención que le permita imponer la importancia que una vez tuvo. 


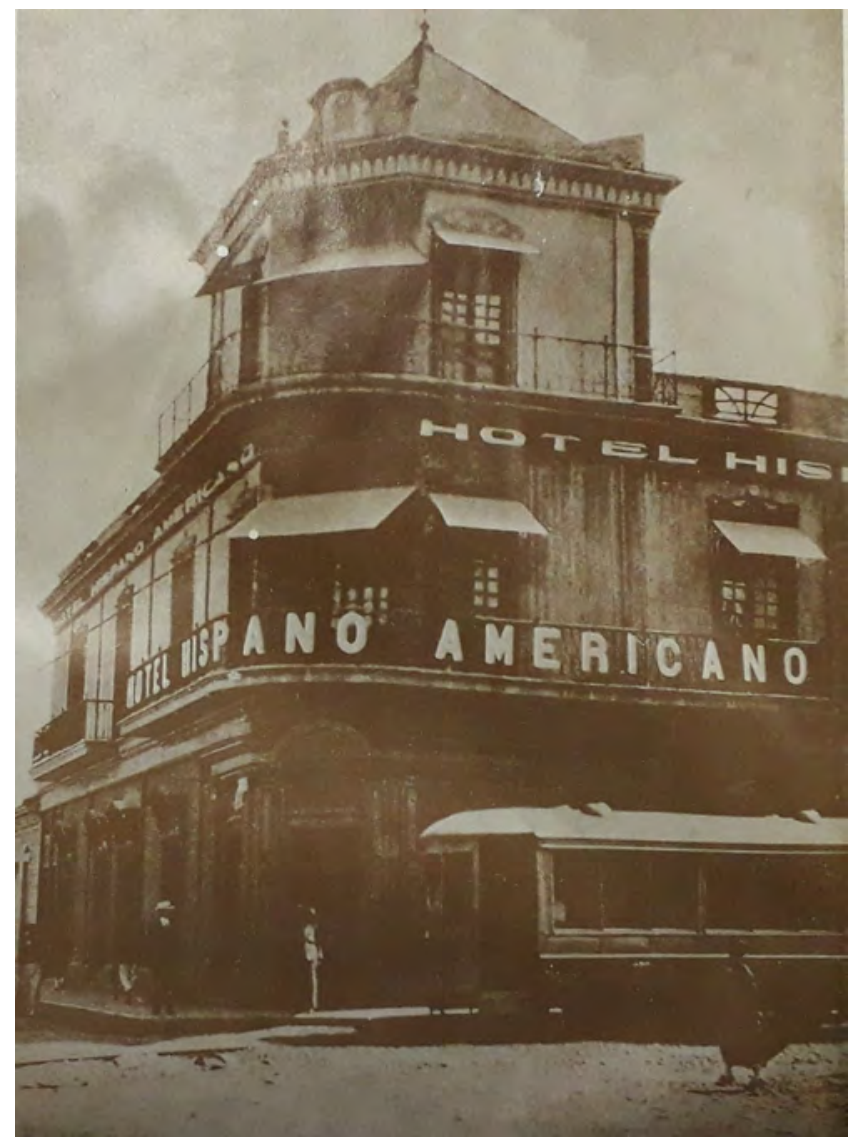

Atrás de la iglesia El Calvario, entre Calle Gerardo Barrios y la 5. avenida Sur, se encontraba la estación de Santa Tecla. Hoy se encuentra aquí el mercado central.

Fotos extraídas del libro: Paseo del recuerdo. San Salvador 1900-1925. 


\section{Referencias bibliográficas}

Aldana. A. (1997). FENADESAL no debe desaparecer. Reportajes, 12 (136), 23-26.

Andino, M. (1927). La obra del gobierno del doctor Quiñonez-Molina (19231927), tomo I. San Salvador, El Salvador: Imprenta Nacional.

Chicas Molina, J. (2015). La vivienda popular en la ciudad de San Salvador: bases para su comprensión historiográfica. AKADEMOS, 2(25), 67-88. Recuperado de https://doi.org/10.5377/akademos.v2i25.4449

Comisión Ejecutiva Portuaria Autónoma [CEPA]. (2014). CEPA y la administración del ferrocarril. 60 años impulsando el progreso del El Salvador (pp. 52-53). San Salvador, El Salvador: CEPA.

Comisión Ejecutiva Portuaria Autónoma [CEPA]. (2018). Memoria de labores junio 2017-mayo 2018. San Salvador, El Salvador: Gobierno de El Salvador.

Comisión Ejecutiva Portuaria Autónoma [CEPA]. (2020). Guía del archivo periférico CEPA-Ferrocarriles Nacionales De El SalvadorFENADESAL. San Salvador, El Salvador: Unidad de Gestión Documental y Archivos (UGDA).

Comisión Ejecutiva Portuaria Autónoma. (s. f.). Reseña histórica de FENADESAL [versión de Adobe Acrobat Reader]. Recuperado de https://www. transparencia.gob.sv/institutions/cepa/documents/390540/download

Decreto del Palacio del Ejecutivo, Diario Oficial, Tomo 39, No. 293, San Salvador (1895).

García, M. Á. (1954). Diccionario histórico enciclopédico de la república de El Salvador. San Salvador desde la conquista hasta el año de 1899. En lo politico, social, ciencias, letras y bellas artes (t. 2) [versión de Adobe Acrobat Reader]. Recuperado de http://repositorios.cihac.fcs.ucr.ac.cr/ cmelendez/handle/123456789/664

La Asamblea Legislativa de la Republica de El Salvador, Acuerdo Ejecutivo No. 269, Diario Oficial, Tomo 247, No. 93, San Salvador (1975).

Leistenschneider, M. y Leistenschneider F. (1980). Gobernantes de El Salvador: biografías. San Salvador, El Salvador. Publicaciones del Ministerio del Interior

Lindo Fuentes, H. (2002). La economía de El Salvador en el siglo XIX. San Salvador, El Salvador: Dirección de Publicaciones e Impresos.

Martin, P. (1985). El Salvador del siglo XX. San Salvador, El Salvador: UCA Editores.

Martínez, N. (2017). La Capital errante. Historia Sísmica de El Salvador [versión de Adobe Acrobat Reader]. Recuperado de http://hdl.handle. net/11298/1001

Ministerio de Educación [MINED]. (2009). Historia 1 y 2 El Salvador (2a ed.) [versión de Adobe Acrobat Reader]. Recuperado de https://sitioinfantil. asamblea.gob.sv/mas/historia-de-el-salvador-tomo-i 
Morrison, A. (07 de noviembre de 2009). Los Tranvías de El Salvador [Mensaje en el blog]. Recuperado de http://www.tramz.com/sv/svs.html

Solorzano, E. (1977). El contrato de concesión de servicio público, terminación $y$ efectos del mismo (Tesis de pregrado). Universidad de El Salvador, San Salvador, El Salvador.

Telegrama, Documentos oficiales, Diario Oficial, Tomo 39, No. 307, San Salvador (1895). Recuperado https://imprentanacional.gob.sv/archivodigital-del-diario-oficial/

Trujillo Vásquez, N. C. (2015). El ferrocarril en El Salvador (1872-1929): conjunto ferroviario sitio del niño, San Juan Opico, La Libertad. Un estudio desde la perspectiva de la arqueología industrial. Un estudio desde la perspectiva de la arqueología industria (tesis de pregrado, Universidad Tecnológica de El Salvador). Recuperado de http:// biblioteca.utec.edu.sv/siab/virtual/tesis/88727.pdf 

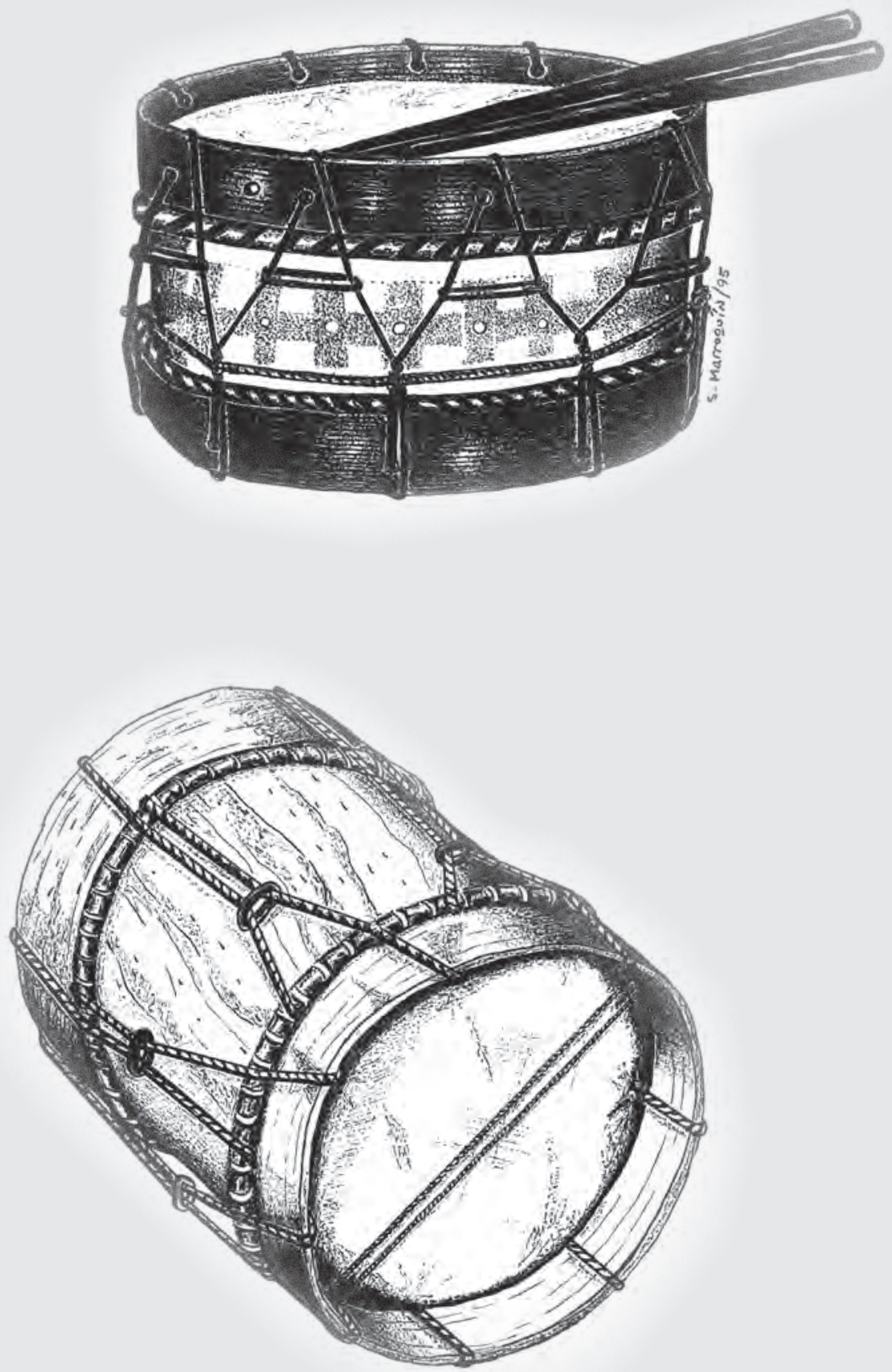

Tambor pequeño o atahualné

Salvador Marroquín 